\title{
SÍNTESE DE ÉSTERES ETÍLICOS A PARTIR DO ÓLEO DE CANOLA
}

\section{R. T. KIFFER ${ }^{1}$, P. A. ARROYO ${ }^{1}$}

${ }^{1}$ Universidade Estadual de Maringá, Departamento de Engenharia Química

E-mail para contato: kiffer.renata@hotmail.com/arroyo@deq.uem.br

\begin{abstract}
RESUMO - O desenvolvimento de combustíveis alternativos a partir de recursos renováveis e a expansão da produção do etanol no Brasil intensificaram o interesse na utilização para a reação de transesterificação. Neste contexto, o cultivo da canola vem ganhando espaço no solo nacional pela alta qualidade do óleo para fins nutricionais e uso industrial. O objetivo do trabalho foi avaliar a influência dos parâmetros velocidade de agitação, tipo e concentração de catalisador básico na transesterificação etílica do óleo de canola refinado. As reações de transesterificação foram realizadas a $60{ }^{\circ} \mathrm{C}$, com razão molar óleo:álcool de 1:6. Os resultados obtidos mostram que as condições ótimas de transesterificação foram obtidas com uma agitação de $800 \mathrm{rpm}$ e utilização do catalisador metilato de sódio, na concentração de $1 \% \mathrm{~m} / \mathrm{m}$. As condições ótimas de reação levaram à obtenção de um rendimento máximo em ésteres de 97,8\%.
\end{abstract}

Palavras-chave: etanol, canola, ácidos graxos, transesterificação, catálise básica.

\section{INTRODUÇÃO}

A maior parte de toda a energia consumida no mundo vem de fontes fósseis (petróleo, carvão e gás natural). No entanto, essas fontes são limitadas e poderá se esgotar num futuro não tão longíncuo, afetando de forma significativa a economia mundial.Deste modo, uma possibilidade para diminuir a dependência dos países pelo petróleo é a produção e utilização de biocombustíveis de primeira geração, que utilizam principalmente óleos vegetais como matéria prima, e de forma crescente os de segunda geração.

Existem muitas espécies vegetais no Brasil que podem ser usadas na produção do biodiesel, tais como a soja, o girassol, o algodão, o amendoim, a mamona, entre outros. No entanto, desde 2008, no que se refere à diversidade no abastecimento de matéria-prima para a produção de biodiesel no Brasil, o óleo de soja permanece em predomínio, com sua utilização variando de 70 a $80 \%$, seguido por sebo bovino e óleo de algodão.

Uma das alternativas de cultura oleaginosa que pode contribuir para a matriz brasileira e que não possui as desvantagens mencionadas do óleo de soja é a canola que apresenta um rendimento médio em óleo entre 34 e $40 \%$, ou seja, mais que 2 vezes o rendimento em óleo da soja. Segundo Tomm (2012), a produção de canola (Brassica napus L.) para fins comerciais e pesquisa se iniciou no Brasil em meados da década de 1970. Em 2013, a área semeada com canola no Brasil foi de 42.168 há e está distribuída 
nos estados do RS $(58,1 \%)$, PR (39,4\%), MS (1,5\%), SP $(0,9 \%)$ e MT $(0,04 \%)$, ou seja, com predominância nos estados do Sul.

\section{MATERIAIS E MÉTODOS}

\subsection{Matéria-prima}

O estudo deste trabalho foi direcionado para a produção de ésteres por meio do óleo de canola refinado. Os reagentes utilizados na reação de transesterificação foi o etanol anidro $(99,8 \%)$ e os catalisadores metilato de sódio (30 \%), $\mathrm{NaOH}$ (P.A) e $\mathrm{KOH}$ (P.A.).

\subsection{Síntese dos Ésteres}

As reações de transesterificação foram realizadas pesando-se $100 \mathrm{~g}$ de óleo de canola em um balão de três bocas, sendo levado a um banho termostático com controle de temperatura $\left(60{ }^{\circ} \mathrm{C}\right)$, sob agitação $(100-1000 \mathrm{rpm})$. Pesou-se o catalisador (solução $30 \%$ de metilato de sódio, $\mathrm{NaOH}$ e $\mathrm{KOH}$ ), na concentração de $1 \%$ em relação à massa de óleo e o álcool (etanol).A quantidade de catalisador foi fixada baseada em estudos publicados na literatura (Meheret al., 2006; Georgogianniet al., 2009), Para cada teste foram preparados doze tubos de ensaio para amostragem, nos tempos de 1, 2, 3, 5, 10, 15,20, 30, 45, 60, 90 e 180 minutos, cada um com $5 \mathrm{~mL}$ da solução de ácido clorídrico $0,1 \mathrm{~mol} / \mathrm{L}$, mantido sob refrigeração.

Após o término da reação, centrifugaram-se os tubos de ensaio para a separação da glicerina na fase aquosa e do biodiesel. Coletou-se aproximadamente $1 \mathrm{~mL}$ do biodiesel e estocou-se cada amostra em um vial. Os vials foram deixados em repouso por um dia na estufa a $60{ }^{\circ} \mathrm{C}$ e, posteriormente, foram colocados em dessecador com sílica acoplado a uma bomba de vácuo, para eliminação do álcool residual, até massa constante.A determinação quantitativa do rendimento dos ésteres no biodiesel foi realizada por cromatografia gasosa, utilizando-se o método EN 14103 e a caracterização conforme normas ANP (2012).

\section{RESULTADOS E DISCUSSÃO}

\subsection{Transesterificação do Óleo de Canola Refinado}

Para o estudo da transesterificação do óleo de canola refinado foi analisada primeiramente a influência do tipo de catalisador, sendo em seguida analisada a influência da quantidade de catalisador e velocidade de agitação no rendimentos em ésteres, e, fixado as variáveis, temperatura e tipo de álcool. 


\section{$\underline{\text { Influência do tipo de catalisador }}$}

Foram analisados três tipos de catalisadores homogêneos na reação de transesterificação, sendo estes o $\mathrm{CH}_{3} \mathrm{ONa}$, o $\mathrm{NaOH}$ e o $\mathrm{KOH}$. As reações ocorreram com $1 \%$ catalisador, razão molar óleo:álcool de 1:6 e temperatura de $60{ }^{\circ} \mathrm{C}$.

A Figura 1 apresenta as curvas de rendimento em ésteres em função dos catalisadores utilizados.

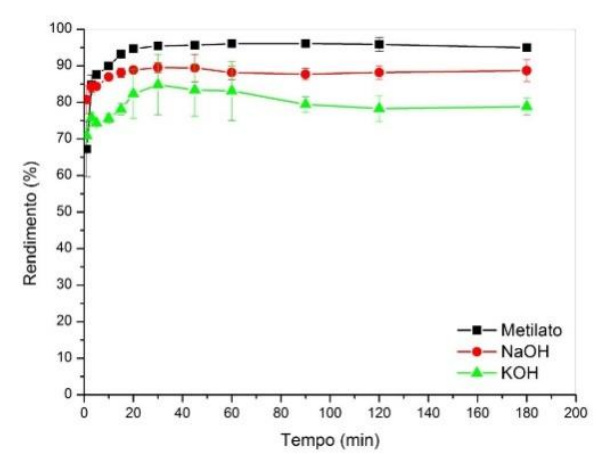

Figura 1 - Rendimento em ésteres em função do tempo, para os catalisadores $\mathrm{CH}_{3} \mathrm{ONa}, \mathrm{NaOH}$ e $\mathrm{KOH}$, com a reação realizada a $60{ }^{\circ} \mathrm{C}, 1 \%$ de catalisador, razão molar óleo:álcool de 1:6 e 800 rpm.

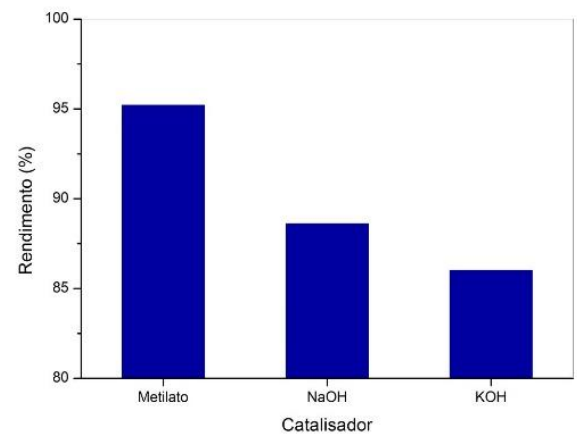

Figura 2 - Rendimento máximo médio em ésteres em função do tipo de catalisador, para reação realizada a $60^{\circ} \mathrm{C}, 1 \%$ de catalisador, razão molar óleo álcool de 1:6 e $800 \mathrm{rpm}$.

Pode-se verificar pelas curvas cinéticas apresentadas na Figura 1 que para todos os catalisadores a condição de equilíbrio é atingida rapidamente, nos primeiros 15 minutos de reação. Além disso, observa-se, um rendimento médio máximo mais alto com o $\mathrm{CH}_{3} \mathrm{ONa}$ e menor para $\mathrm{KOH}$. Para os catalisadores $\mathrm{NaOH}$ e $\mathrm{KOH}$, após ter obtido equilíbrio em 15 minutos, observa que há uma queda no rendimento em ésteres com o tempo de reação, que pode ser atribuído a uma emulsificação maior do meio reacional, ou seja, dos ésteres etílicos com os intermediários mono e diacilglicerídeos ou sabões formados (Freedmanet al., 1985). Isto praticamente não ocorreu com a utilização de $\mathrm{CH}_{3} \mathrm{ONa}$, provavelmente pela maior reatividade do etóxido proveniente da adição deste catalisador. Para os demais catalisadores estudados o etóxido só é formado no meio pela reação do etanol com o hidróxido, o que contribui para a menor velocidade de reação observada.

Portanto, o desempenho catalítico do $\mathrm{CH}_{3} \mathrm{ONa}$ foi melhor, apontando para um maior rendimento máximo médio, que por sua vez foi maior do que os obtidos com $\mathrm{NaOH}$ e $\mathrm{KOH}$, respectivamente, na reação de transesterificação do óleo de canola refinado. 
A Figura 2 mostra o rendimento máximo médio em ésteres em função do tipo de catalisador estudado, ressaltando o melhor desempenho alcançado com a utilização de metilato de sódio $\left(\mathrm{CH}_{3} \mathrm{ONa}\right)$.

\section{$\underline{\text { Influência da quantidade de catalisador }}$}

No estudo da influência da quantidade de catalisador $(0,5,1,0,1,5,2,0 \%)$ sobre o rendimento em ésteres, as reações ocorreram com razão molar óleo:álcool de1:6, temperatura de $60{ }^{\circ} \mathrm{C}$, velocidade de agitação de $800 \mathrm{rpm}$ e catalisador $\mathrm{CH}_{3} \mathrm{ONa}$. A Figura 3 apresenta as curvas de rendimento em ésteres em função do tempo de reação para cada concentração de $\mathrm{CH}_{3} \mathrm{ONa}$ utilizada.

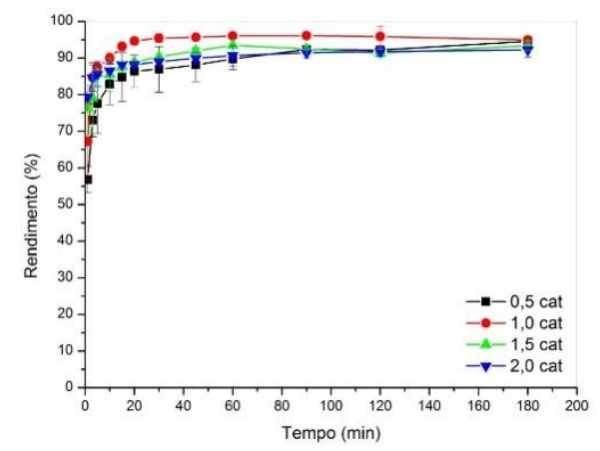

Figura 3 - Rendimento em ésteres em função do tempo, para as diferentes quantidades de catalisador $\mathrm{CH}_{3} \mathrm{ONa}$ utilizadas na reação a $60^{\circ} \mathrm{C}$, razão molar óleo:álcool de 1:6 e 800 rpm

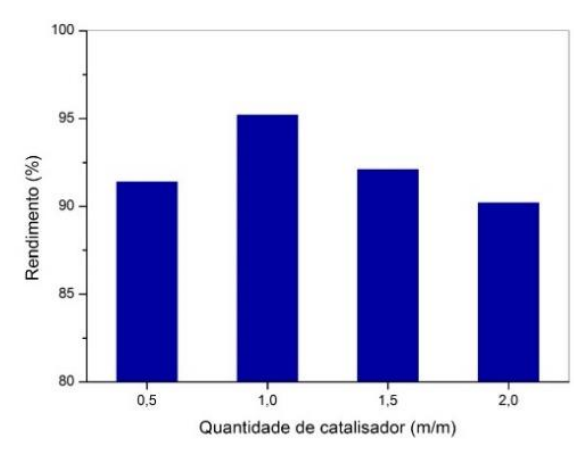

Figura 4 - Rendimento máximo médio em ésteres em função da quantidade de $\mathrm{CH}_{3} \mathrm{ONa}$, para reação realizada a $60^{\circ} \mathrm{C}$, razão molar óleo:álcool de 1:6 e 800 rpm

Pelas curvas cinéticas mostradas na Figura 3 é possível observar que a menor quantidade de catalisador utilizada $(0,5 \%)$ leva a um rendimento em ésteres relativamente menor e em tempo de reação maior para se atingir o equilíbrio no meio reacional. Quando a quantidade de catalisador aumentada para $1 \%$ a velocidade de reação também aumenta, pois o equilíbrio no meio reacional é atingido em tempos menores. Embora o rendimento em ésteres alcançado para um tempo longo de reação de 180 minutos seja muito próximo para toas as quantidades de catalisador utilizada, o rendimento máximo médio em ésteres no período é influenciado pela quantidade de catalisador utilizada na reação de transesterificação, ressaltando a influência da quantidade de catalisador na velocidade de reação. 
Nas mesmas condições Ma e Hanna (1998) obtiveram um rendimento em ésteres próximo a $80 \%$ depois de 1 minuto de reação para o óleo de soja, canola, e girassol e, após 1 hora, o rendimento em ésteres foi quase o mesmo para todos os óleos estudados milho, canola, amendoim, girassol, palma e soja (93 - 98\%), em concordância com valores apresentados na Figura 4. Outros autores também relataram o aumento no rendimento em ésteres com o aumento da quantidade de catalisadores (Georgogianniet al., 2009 e Freedmanet al., 1984).

Em concordância com os resultados obtidos a literatura relata que a concentração de $1 \%$ de catalisador leva à obtenção de rendimentos mais altos em éster na transesterificação alcalina de óleos vegetais (Meheret al., 2006; Georgogianniet al., 2009), Também foi observado na literatura que a utilização de quantidades mais altas de catalisador, em temperatura e razão molar óleo:álcool elevadas, tendem a favorecer uma emulsificação do éster formado, levando a uma diminuição do rendimento médio em ésteres observado, em concordância com os dados apresentado na Figura 4. De fato, Vicente et al. (2004) e Knotheet $a l$. , (2006) propõem que o excesso de catalisador alcalino na reação de transesterificação tende a saponificar o triacilglicerídeo ou promover a emulsão do éster formado com o glicerol, acarretando diminuição da conversão em ésteres.

\section{Influência da velocidade agitação}

A Figura 5 mostra as curvas de rendimento em ésteres em função da velocidade de agitação, para as reações realizadas a $60{ }^{\circ} \mathrm{C}, 1 \%$ de $\mathrm{CH}_{3} \mathrm{ONa}$ e razão molar óleo:álcool de 1:6.

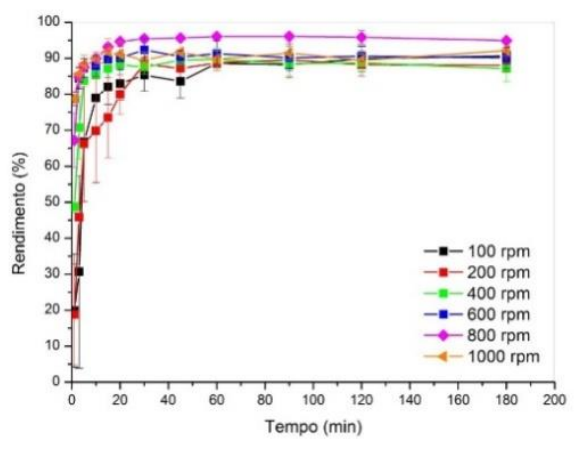

Figura 5 -Rendimento em ésteres em função da velocidade de agitação, para a reação realizada a $60{ }^{\circ} \mathrm{C}, 1 \% \mathrm{CH}_{3} \mathrm{ONa}$ e razão molar óleo:álcool de 1:6 .

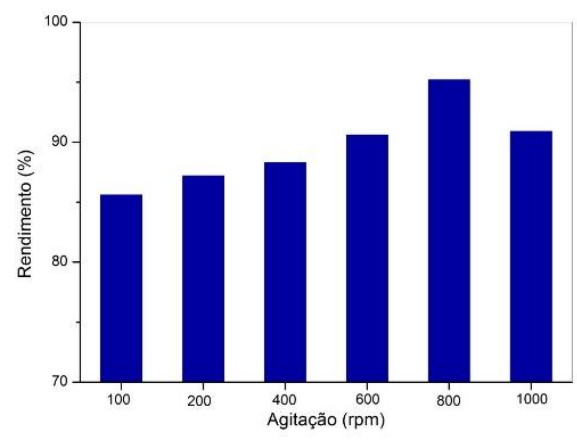

Figura 6 - Rendimento Máximo médio em ésteres em função da velocidade de agitação, para a reação realizada a $60^{\circ} \mathrm{C}$, $1 \% \mathrm{CH}_{3} \mathrm{ONa}$ e razão molar óleo:álcool de 1:6.

Pelas curvas apresentadas na Figura 5 e pelos valores de rendimento máximo médio em ésteres mostrado na Figura 6 pode-se observar que a agitação do meio reacional influência 
de forma significativa a transferência de massa na reação de transesterificação do óleo de canola com etanol, pois o meio é composto por fases imiscíveis, necessitando do contato íntimo entre as fases para que a reação ocorra. Assim, é possível observar que em velocidades de agitação mais baixas o tempo necessário para se atingir a condição de equilíbrio no sistema é mais alto (60 min), indicando uma velocidade de reação menor, possivelmente devido a uma menor taxa de transferência de massa no sistema reacional nestas condições.

Quando a velocidade de agitação é aumentada para $800 \mathrm{rpm}$ o tempo necessário para se atingir o equilíbrio diminui de forma significativa (15 min), indicando uma menor resistência à transferência de massa no meio, levando a uma velocidade de reação maior e, também, a um rendimento em ésteres mais alto (95,2\%). Para velocidade de agitação mais alta (1000 rpm), embora o tempo para se atingir a condição de equilíbrio seja a mesma que em $800 \mathrm{rpm}$, o rendimento em ésteres se mostrou menor. Isto pode ser devido a uma maior intensidade de mistura no meio reacional, acarretando um nível maior de emulsificação, já que o etanol também pode contribuir para isto, o que leva à diminuição do rendimento em ésteres $(90,9 \%)$. De fato, Noureddini et al. (1998) também verificaram que ao utilizar velocidades de agitação muito altas a conversão de óleo diminuía, atribuindo este efeito a uma possível mistura do glicerol com o álcool e os outros reagentes.

\section{Caracterização dos ésteres obtidos na transesterificação do óleo de canola}

Utilizando-se as condições ótimas para a reação de transesterificação do óleo de canola refinado obtidas no presente estudo, realizaram-se dois ensaios (réplicas), a fim de se obter uma quantidade de ésteres suficiente para realizar as análises de qualidade do biodiesel obtido. Além disso, os ensaios também podem ser utilizados para análise da reprodutibilidade do sistema reacional. Os resultados de rendimento em ésteres em função do tempo de reação em condições são mostrados na Figura 7 e resultado de características de qualidade na Tabela1.

A curva apresentada na Figura 7 mostra que o erro associado aos valores de rendimento em ésteres é pequeno, indicando a boa reprodutibilidade do sistema reacional. Também é possível observar rendimento em ésteres superior a $95 \%$ em tempos de reação de aproximadamente 15 a 20 minutos, em concordância com os resultados observados anteriormente.

Os ésteres obtidos e purificados foram caracterizados conforme mostra a Tabela 1, para avaliação da qualidade do biodiesel produzido. 


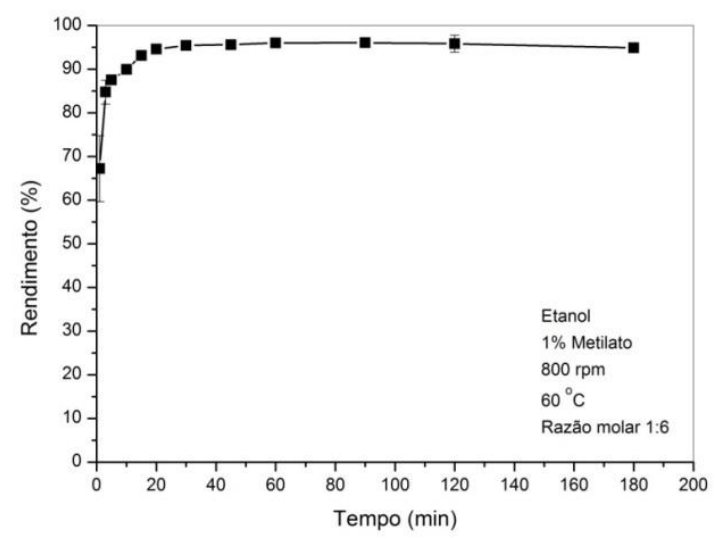

Figura 7 - Rendimento em ésteres em função do tempo, para as condições ótimas de reação de transesterificação do óleo de canola refinado: temperatura de $60{ }^{\circ} \mathrm{C}, 1 \% \mathrm{CH}_{3} \mathrm{ONa}$, razão molar óleo:álcool de1:6 e 800 rpm.

Tabela 1- Resultados de características de qualidade do éster etílico obtido a $60{ }^{\circ} \mathrm{C}, 1 \%$ de metilato de sódio, razão molar 1:6 e $800 \mathrm{rpm}$.

\begin{tabular}{lcccc}
\hline \multicolumn{1}{c}{ Item Analítico } & Métodos & Especificação & Unidade & Resultados \\
\hline Massa Específica a 20 ${ }^{\circ} \mathrm{C}$ & ASTM D 4052 & $850,0-900,0$ & $\mathrm{~kg} / \mathrm{m}^{3}$ & $\mathbf{8 7 5 , 1}$ \\
Viscosidade Cinemática a $40{ }^{\circ} \mathrm{C}$ & ASTM D 445 & $3,000-6,000$ & $\mathrm{~mm} / \mathrm{s}$ & $\mathbf{4 , 7 9 0}$ \\
Teor de água & ASTM D 6304 & máx. 0,020 & $\mathrm{g} / 100 \mathrm{~g}$ & $\mathbf{0 , 0 3 1}$ \\
Ponto de Fulgor & ASTM D 93 & mín. 100,0 & ${ }^{\circ} \mathrm{C}$ & $>\mathbf{1 6 0}$ \\
Resíduo de Carbono & ASTM D 4530 & máx. 0,05 & $\mathrm{g} / 100 \mathrm{~g}$ & $\mathbf{0 , 0 1}$ \\
Enxofre Total & ASTM D 5453 & máx. 10,0 & $\mathrm{mg} / \mathrm{kg}$ & $\mathbf{5 , 5}$ \\
Sódio + Potássio & NBR 15553 & máx. 5,0 & $\mathrm{mg} / \mathrm{kg}$ & $\mathbf{2 , 1}$ \\
Cálcio + Magnésio & NBR 15553 & máx. 5,0 & $\mathrm{mg} / \mathrm{kg}$ & $\mathbf{0 , 7}$ \\
Fósforo & NBR 15553 & máx. 10,0 & $\mathrm{mg} / \mathrm{kg}$ & $\mathbf{0 , 1}$ \\
\hline
\end{tabular}

Os resultados encontrados para as características analisadas estão de acordo com as especificações ANP para padrões de combustível biodiesel. Os resultados das características analisadas podem estar relacionados com a composição dos ácidos graxos do óleo de canola refinado (Knotheet al., 2005; Siemens e Daun, 2005).

\section{CONCLUSÕES}

De maneira geral, o rendimento em ésteres aumenta rapidamente nos primeiros 5 minutos de reação, atingindo uma condição de equilíbrio em tempos relativamente curtos (15-45 
minutos). $\mathrm{O}$ aumento da temperatura faz com que este equilíbrio seja alcançado em tempos menores.

A utilização do catalisador metilato de sódio na concentração $1 \%$ para a transesterificação etílica do óleo de canola levou a um aumento no rendimento da reação, em relação à utilização de $\mathrm{NaOH}$ e KOH. Verificou-se também que a velocidade de agitação de 800 rpm influencioude forma positiva a reação contribuindo para o aumento do rendimento.

\section{REFERÊNCIAS}

1. ANP - Agência Nacional do Petróleo, Gás Natural e Biocombustíveis. Resolução ANP No 14, de 11.5.2012 - DOU 18.5.2012.

2.FREEDMAN, B.; PRYDE, E. H.; MOUNTS, T. L. Variables affecting the yield of fatty esters transesterified vegetable oils. J. Am. Oil Chem. Soc., v. 61, n. 10, p. 1638-1643, 1984.

3.GEORGOGIANNI, A. K.; KATSOULIDIS, P.J.; POMONIS, G.; MANOS, M.G. Transesterification of rapeseed oil for the production of biodiesel using homogeneous and heterogeneous catalysis. FuelProcessing Technology, v. 90, 1016 - 1022, 2009.

4. KNOTHE G.; BAGBY, M.O.; RYAN, T.W. Dependence of biodiesel fuel properties on the structure of fatty acid alkyl esters, Fuel Process Technol, v.86, p. 1059-70, 2005.

5. MA, F., HANNA, M. A., 1999, "Biodiesel production: a review", Bioresource Technology, V. 70, pp. 1-15, 1999.

6.MEHER, L. C.; DHARMAGADDA, V. S. S.; NAIK, S. N. Optimization of alkalicatalizedtransesterification of Pongamiapinnata oil for production of biodiesel. Bioresource Technology, v. 97, p. 1392-1397, 2006

7. NOUREDDINI, H., HARKEY, D., MEDIKONDURU, V. A. A Continuous Process for the Conversion of Vegetable Oils into Methyl Esters of Fatty Acids, J. Am. Oil Chem. Soc. V. 75, p.1775-1783, 1998.

8. SIEMENS, B.J and DAUN, J.K. Determination of the Fatty Acid Composition of Canola, Flax, and Solin by Near-Infrared Spectroscopy. JAOCS, v.82 p. 153-157, 2005.

9.TOMM, G. O.; KUTCHER, H. R.; EASTON, A. Eradication of Blackleg disease of canola in Brasil - A sucess story. In: International Crop Science Congress, 2012, Bento Gonçalves, International Crop Science Society, 2012. Poster presentation- Agronomy, Resumo 3191 